\title{
Amenazas externas y Política de Defensa de Japón. Las modificaciones del gobierno de Abe $^{1}$
}

\author{
External threats and Japan's defense policy. The \\ changed made by the Abe administration.
}

Marina Zalazar ${ }^{2}$

Resumen: La política de defensa nipona es un caso particular de estudio por las limitaciones impuestas al país asiático luego de la Segunda Guerra Mundial. Estas condiciones presentes en el artículo 9 de la constitución continúan vigentes al día de hoy, aunque se las ha reinterpretado a lo largo de los años para que Japón amplíe cada vez más sus capacidades defensivas.

El primer ministro actual, Shinzo Abe, es quien ha introducido las modificaciones más importantes, que serán analizadas teniendo en cuenta nociones del realismo neoclásico para sostener la hipótesis de que las mismas han sido adoptadas en función de amenazas provenientes de China y Corea del Norte y de las presiones de Estados Unidos sobre el gobierno japonés.

Palabras clave: Defensa, Japón, China, Corea del Norte, Estados Unidos, Shinzo Abe.

Abstract: The Japanese defence policy is a special case study because of the limitations imposed after World War 2 on this Asian country. These limitations, detailed in article 9 of the national constitution, are still valid; however, they have been reinterpreted iso as to widen Japan's defensive capabilities.

The most important changes have been introduced by current Prime Minister, Shinzo Abe, and they will be analyzed following the ideas of neoclassical realism so as to support the hypothesis that these changes respond to perceived threats from China and North Korea, and also to the U.S. pressure on Japanese government.

Key words: Defense, Japan, China, North Korea, United States, Shinzo Abe.

\footnotetext{
${ }^{1}$ Recibido: 30/07/2018. Aceptado: 27/08/2018

${ }^{2}$ Licenciada en Relaciones Internacionales. Docente adscripta en la cátedra de Geografía I de la Lic. en Turismo y docente colaboradora en la cátedra de Política Internacional de la Lic. en Relaciones Internacionales, ambas en la UNR. Estudiante de la Maestría de Integración y Cooperación Internacional del CERIR de la UNR. Email: marina.zalazar@hotmail.com
} 


\section{Introducción}

La política de defensa nipona es un caso particular de estudio por las limitaciones impuestas al país asiático luego de la Segunda Guerra Mundial. La ocupación estadounidense entre 1945 y 1952 obligó a Japón a adoptar una nueva constitución que se adaptase a las normas y valores occidentales, pero además impuso al país nipón la prohibición de recurrir a la guerra o al uso de la fuerza en sus relaciones internacionales, por lo que no debía mantener un ejército regular, ni potencial bélico.

Estas condiciones, contenidas en el artículo 9 de la constitución ${ }^{3}$, continúan vigentes al día de hoy, aunque se las ha reinterpretado a lo largo de los años para que Japón amplíe cada vez más sus capacidades defensivas. Es por esto que las modificaciones introducidas a la política de defensa nipona, desde que en 1954 son creadas las Fuerzas de Autodefensa (FAD) ${ }^{4}$, forman parte de un proceso, de un movimiento progresivo que tiene como objetivo la normalización de la misma (López Vidal, 2007, p.191)

Los cambios que trajo consigo el siglo XXI, como: la aparición de la lucha contra el terrorismo en la agenda global, la crisis económica del 2008 que afecta a los países avanzados, la confirmación de China como potencia global, las amenazas nucleares de Irán y Corea del Norte; han acelerado este proceso de modificación de la política de defensa nipona, más que nada de la mano del primer ministro Abe.

El presente trabajo, buscará analizar las modificaciones introducidas durante el gobierno de Abe para confirmar la hipótesis de que las mismas son producto de amenazas externas que percibe el gobierno japonés de parte de China y Corea del Norte, y de las presiones de Estados Unidos sobre el gobierno nipón. Es por esto que el recorte temporal del análisis comienza en 2012 cuando Abe asume su segundo mandato como primer ministro y termina en 2015, cuando asume Trump la presidencia en Estados Unidos, lo que abrió un período de gran incertidumbre internacional que complejiza el análisis y excedería la extensión del trabajo.

Para analizar de forma acabada las modificaciones se utilizarán algunas nociones teóricas del realismo neoclásico que entiende que la política exterior de un estado es determinada en primera instancia por presiones sistémicas, por la estructura del escena-

\footnotetext{
3 “Aspirando sinceramente a una paz internacional basada en la justicia y el orden, el pueblo japonés renuncia para siempre a la guerra como derecho soberano de la nación y a la amenaza o al uso de la fuerza como medio de solución en disputas internacionales. Con el objeto de llevar a cabo el deseo expresado en el párrafo precedente, no se mantendrán en lo sucesivo fuerzas de tierra, mar o aire como tampoco otro potencial bélico. El derecho de beligerancia del estado no será reconocido". Artículo 9 de la Constitución de Japón, 1947. Disponible online en: https://webjapan.org/factsheet/es/pdf/es09 constitution.pdf

${ }^{4}$ Las FAD son administradas, desde 2007, por el Ministerio de Defensa de Japón (antes estaban bajo la órbita de la ya disuelta Agencia de Defensa). Similar a otras fuerzas armadas, están conformadas por 3 cuerpos: la fuerza aérea, la fuerza marítima y la fuerza terrestre. Desde el 2014 están comandadas por el Almirante Katsutoshi Kawano. Según un informe del Instituto Internacional de Estudios Estratégicos (2017) las FAD tienen un personal de 300.000 (trescientas mil) personas, entre personal activo y de reserva, y cuentan con equipamiento militar de avanzada, como por ejemplo 3 barcos portaaviones, más de 600 aviones preparados para combate, 33 barcos destructores, 19 submarinos de combate. Posee además variedad de misiles SAM (superficie-aire), ASM (aire-superficie) y AAM (aire-aire) de corto y medio alcance. Su fuerza marítima es tal vez la más equipada de las 3, teniendo en cuenta el extenso litoral marítimo de Japón. Su principal proveedor de armas es su principal aliado, Estados Unidos.
} 
rio internacional; pero además que esas presiones sistémicas tienen que ser interpretadas por actores al nivel de la unidad, es decir, por los que toman decisiones en política exterior y de defensa (Rose, 1998) Es así, que las percepciones se conforman como elemento de importancia para el realismo neoclásico, y por lo tanto los que toman decisiones en política exterior y de defensa tienen un rol central para esta corriente, dado que están ubicados en la intersección entre lo internacional y lo doméstico (Lobell, 2009, p.43), son el cable conductor a través del cual las variables estructurales se traducen en política exterior (Feaver, 2000, p.81) Otro de los elementos centrales del análisis del realismo neoclásico es el impacto del poder relativo en la política exterior y de defensa y las percepciones que los que toman decisiones en política exterior tengan sobre ese poder relativo. Rose $(1998$, p.167) sostiene que para esta teoría un aumento en el poder material relativo puede llevar a la ampliación de la ambición y enfoque de las actividades de política exterior de un estado; pero el grado con el que esto suceda va a depender de las percepciones de los que toman las decisiones.

Las percepciones japonesas en torno a las amenazas norcoreana y china, y en torno a las presiones estadounidenses serán tomadas de discursos de funcionarios de alto nivel, como el primer ministro o ministros de asuntos externos, y de publicaciones oficiales de los ministerios de defensa y asuntos externos. Dado que la mayor parte de estos documentos están disponibles sólo en idioma inglés, se proporcionará una traducción al español elaborada por la autora en las referencias al pie de página.

\section{Los ajustes a la política de defensa en el gobierno de Abe}

Una de las prioridades en política exterior del Primer Ministro Abe ha sido la modificación de la política de defensa basada en el artículo 9 de la constitución nacional. El corolario de este objetivo fueron 2 leyes, impulsadas por Abe, que fueron aprobadas en 2015: "Ley de enmiendas parciales a la ley de las Fuerzas de Autodefensa y otras leyes existentes que garantizan la paz y seguridad de Japón y la comunidad internacional" y "Ley de actividades de cooperación y apoyo a las fuerzas armadas de otros países en situaciones dónde la comunidad internacional actúe colectivamente para la paz y la seguridad"

Estas leyes son el resultado de modificaciones previas que se venían realizando desde que Abe asume en el año 2012. Una de sus primeras medidas en el área de la defensa fue la creación del Consejo de Seguridad Nacional (NSC) en 2013. Según el Ministerio de Asuntos Externos, el Consejo abordará diversas temáticas de seguridad nacional, bajo un fuerte liderazgo del Primer Ministro (MOFA, 2014b), lo que según Berkshire Miller (2014b) permitirá una dirección más eficiente del ejecutivo japonés sobre la política de defensa. EI NSC funciona a partir de la llamada "reunión de 4 ministros", en la cual participan el Primer Ministro, el Jefe de Gabinete, el Ministro de Asuntos Externos y el Ministro de Defensa; y está conformado más que nada por burócratas y no necesariamente por especialistas del área, lo que el autor antes mencionado considera como insostenible en el largo plazo (Berkshire Miller, 2014b)

Otra de las modificaciones de envergadura se relaciona con la relajación de la prohibición de exportación de armas, vigente desde 1967 y en concordancia con el artículo 9 que sostenía que Japón no podría tener "potencial bélico". En el 2014 se modifica 
esta prohibición para habilitar la exportación de equipamiento militar a países aliados que acepten no vender este equipamiento a terceros sin antes contar con la aprobación del gobierno japonés. Esta medida del gobierno de Abe no sólo responde a la búsqueda de una mayor capacidad de desarrollar la industria armamentística nipona, sino también a la presión de grandes corporaciones japonesas que buscan ampliar el mercado. De todos modos, la industria militar japonesa está todavía lejos de ser competitiva a nivel internacional y tiene todavía que recorrer un largo camino para desarrollarse.

La primera decisión que anticipa lo que luego serían las leyes del 2015, fue la Decisión del Gabinete de julio de 2014, en la cual se realiza una reinterpretación del artículo 9 a favor de la defensa colectiva. En esta decisión, el gobierno de Abe expresó que el uso de la fuerza por parte de las FAD que permitiría la constitución, es el ejercicio de acciones de defensa propia para mantener la paz y la seguridad de Japón y asegurar su supervivencia. El gobierno de Abe basó esta interpretación en el preámbulo de la constitución y en su artículo 13 que estipulan el derecho de los ciudadanos japoneses a la vida, la libertad, al logro de la felicidad y a vivir en paz (MOFA, 2014)

Esta reinterpretación fue luego recogida por las Nuevas Directrices para la alianza nipo-estadounidense que se adoptaron a principios de 2015, como puede leerse en el documento aprobado por ambos gobiernos:

The Self-Defense Forces will conduct appropriate operations involving the use of force to respond to situations where an armed attack against a foreign country that is in a close relationship with Japan occurs and as a result, threatens Japan's survival and poses a clear danger to overturn fundamentally its people's right to life, liberty, and pursuit of happiness, to ensure Japan's survival, and to protect its people" ${ }^{5}$ (MOD, 2015, p.16)

Las nuevas directrices preveían el caso de que Japón necesitase utilizar el uso de la fuerza y daban así el visto bueno a las leyes que Abe estaba intentando aprobar en la Dieta. Otra cuestión interesante que puede leerse en las directrices es que las situaciones que amenazan la paz y la seguridad de Japón no pueden ser definidas geográficamente y que debido al complejo ambiente de seguridad, ambos gobiernos tomarán medidas para asegurar la paz y la seguridad de Japón, incluso cuando un ataque contra el país asiático no haya ocurrido, estando entonces en clara sintonía con la nueva legislación que se estaba debatiendo. (MOD, 2015, p.4,7)

Llegamos entonces a las dos leyes aprobadas en septiembre de 2015. La primera ley, mencionada al principio de este apartado, amplía las actividades que las FAD pueden realizar y agrega la situación en la cual un estado con una relación cercana a Japón es atacado y ese ataque amenaza la supervivencia y el derecho de los ciudadanos japoneses a la vida, la libertad y el logro de la felicidad. La idea general es reforzar la cooperación de las FAD con aquellos estados que estén realizando actividades para defender a Japón y en ese sentido se aclara que el personal de las FAD puede hacer uso de armas, estando en servicio, cuando su vida se vea amenazada; como así también cuando la vida del per-

\footnotetext{
5 "Las Fuerzas de Autodefensa llevarán a cabo operaciones que involucren el uso de la fuerza para responder a situaciones en que ocurra un ataque armado contra un país extranjero que tenga relaciones cercanas con Japón y como resultado del mismo, se vea amenazada la supervivencia de Japón y se ponga en peligro el derecho del pueblo japonés a la vida, la libertad y la felicidad, para así garantizar la supervivencia de Japón y proteger a sus ciudadanos."
} 
sonal de las Fuerzas Armadas de Estados Unidos y otros estados se vea amenazada al realizar operaciones que contribuyan a la defensa de Japón. También, se amplían las actividades que las FAD pueden realizar en carácter de apoyo logístico.

Por otro lado, la segunda ley mencionada hace referencia a las actividades de cooperación y apoyo que las FAD pueden realizar cuando la comunidad internacional, de acuerdo a la Carta de Naciones Unidas, esté combatiendo una amenaza a la paz y la seguridad internacional. Estas actividades de las FAD son consideradas como contribuciones independientes y proactivas de Japón como un miembro responsable de la comunidad internacional.

La nueva legislación de seguridad, considera que Japón puede hacer uso de la fuer$z a$, a través de sus FAD, cuando se cumplan una serie de condiciones: cuando ocurre un ataque armado contra Japón o contra un estado que tiene relaciones cercanas a Japón y esto amenaza la supervivencia de Japón y pone en peligro el derecho de sus ciudadanos a la vida, la libertad y el logro de la felicidad. Este uso de la fuerza será siempre ejercido de acuerdo a las leyes internacionales, lo que podría incluir el ejercicio de la autodefensa colectiva; cuando no hay otros medios disponibles para repeler el ataque; y el uso de la fuerza será limitado al mínimo necesario.

Esta nueva legislación es el corolario de la nueva política de defensa de Abe que es denominada como "pacifismo proactivo" Como bien lo dice este nombre, Japón no abandona el pacifismo del artículo 9 de su constitución, sino que lo reinterpreta en favor de la defensa de su supervivencia e integridad. De ninguna manera estas leyes permiten a Japón usar la fuerza como medio para resolver conflictos, sino que consideran al uso de la fuerza sólo en el caso de que la supervivencia de Japón y la integridad de sus habitantes se vean amenazadas. Por otro lado, las FAD siguen siendo Fuerzas de Autodefensa y no fuerzas militares, y continúan teniendo restricciones a la hora de participar en actividades internacionales.

\section{La política de defensa de Abe y las amenazas externas}

¿Por qué Japón ha agilizado su proceso de normalización de la política de defensa? ¿A qué responden las modificaciones de Abe introducidas en este ámbito? El realismo neoclásico puede ayudarnos a responder estas preguntas a partir de su noción de percepciones de amenaza provenientes del sistema internacional y del ámbito regional. Los que interpretan estas variables provenientes del exterior como amenazantes/preocupantes o no son los hacedores de política, los funcionarios involucrados en la definición de la política exterior y de defensa; de ahí que sea crucial revisar las publicaciones oficiales de ministerios japoneses, como así también discursos de funcionarios para identificar cuáles son las amenazas y preocupaciones de Japón que fomentaron los ajustes en su defensa.

Comenzando por el ambiente regional, en el Libro Blanco ${ }^{6}$ para el año 2016, el Ministerio de Defensa dedica un capítulo a explicar la nueva legislación de seguridad y sus

\footnotetext{
${ }^{6}$ El Libro Blanco se publica de manera anual por el Ministerio de Defensa y a principios del año; tomando el período que va de Julio del año anterior a Mayo del año corriente. Dado que la nueva legislación fue aprobada en Septiembre de 2015, el Libro Blanco de 2016 es el que contiene la descripción más acabada
} 
porqués. En el mismo se puede leer la preocupación de Japón por el ambiente que lo rodea y la directa relación con la nueva legislación:

The security environment surrounding Japan is increasingly severe, as seen in the shift in the global power balance, development and proliferation of weapons of mass destruction and ballistic missiles, and threats such as international terrorism. [...] No country can mantain its own security only by itself. [...] It is essential to prevent threats from reaching Japan by appropriately developing Japan's own capabilities, further elevating the effectiveness of the Japan-United States Security Arrangements and enhancing the deterrence of the Japan-United States Alliance."7 (Mod, 2016, p.210)

En concreto, las mayores preocupaciones de Japón con respecto al ambiente regional tienen que ver con Corea del Norte y China. Con respecto a China, el entonces Ministro de Defensa Gen Nakatani, mencionaba en el Libro Blanco 2015 (MOD, 2015) una serie de asuntos que agravaban el entorno de seguridad japonés: aumento del presupuesto de defensa en más del $10 \%$ por 5 años consecutivos; incursiones de barcos chinos en aguas territoriales niponas; sobrevuelo de aviones caza chinos en las cercanías de aviones de las FAD; y falta de transparencia en sus asuntos militares y en cuestiones de seguridad. A estas cuestiones hay que sumarle la histórica disputa entre ambos estados por las islas Senkaku y el ascenso chino en los asuntos mundiales que cambia el balance de poder no sólo en la región Asia-Pacífico, sino también en el mundo.

Aunque China no sea mencionada como una amenaza de manera expresa ${ }^{8}$, las actividades que realiza en los mares circundantes a Japón y su desarrollo militar son mencionados en documentos oficiales como un desafío para la seguridad del país nipón. Por ejemplo en el Libro Blanco 2016, se puede leer como las actividades de China en el Mar de China Oriental y la falta de transparencia en el refuerzo de sus capacidades militares son una preocupación para la seguridad de Japón:

“[...] as China's reinforcement of its military capabilities without transparency, along with active maritime advancement, rapidly shift the regional military balance, China's attempts to change the status quo in the East and South China Seas based on its own assertions as well as its risk of causing unintended consequences due to misunderstanding or miscalculation have become security concerns to the region including Japan and to the international community" ${ }^{9}$ (MOD, 2016)

de la legislación. De todos modos, Libros Blancos de años anteriores son tenidos en consideración para evaluar el ambiente de seguridad de Japón.

\footnotetext{
7 "El ambiente de seguridad que circunda a Japón se está volviendo cada vez más severo, como se puede observar en el cambio en el balance de poder global, en el desarrollo y proliferación de armas de destrucción masiva y misiles balísticos, y en amenazas como el terrorismo internacional. [...] Ningún país puede mantener su seguridad por sí solo. [..] Es esencial prevenir la llegada de estas amenazas a Japón, desarroIlando de manera apropiadas las capacidades de Japón, elevando la efectividad de los Acuerdos de Seguridad nipo-estadounidenses y reforzando la capacidad de disuasión de la Alianza Japón - Estados Unidos."

${ }^{8}$ El Primer Ministro Abe y su Ministro de Relaciones Exteriores Fumio Kishida hacen referencia a la relación que se persigue con China como una "Relación Estratégica Mutualmente Beneficiosa"; a pesar de las preocupaciones que generan las actividades de China y teniendo en cuenta su interdependencia económica.

9 "[...] mientras el refuerzo de China de sus capacidades militares sin transparencia, junto con un avance marítimo activo cambian rápidamente el balance militar regional, los intentos de China de cambiar el status quo en los Mares del Este y del Sur de China siguiendo sus propias reivindicaciones, como así también el riesgo que esto conlleva de causar consecuencias no intencionadas debido a malentendidos o
} 
No sólo en documentos relacionados con la defensa de Japón encontramos referencias a la preocupación por China. En el Libro Azul ${ }^{10}$ 2016, previo a la enumeración de las actividades chinas en el área circundante a Japón, se encuentra la siguiente referencia: "[...] a series of military developments in China, as seen prominently in recent years, raises concern in the region and the entire international community" ${ }^{11}$ (MOFA, 2016, p.3) Incluso el Primer Ministro Abe se refirió con preocupación a las actividades chinas en la región, durante una entrevista concedida a The Wall Street Journal en el 2013: "There are concerns that China is attempting to change the status quo by force, rather than by rule of law." ${ }^{12}$ (Baker y Nishiyama, 2013)

Para el caso chino, las variables externas que guían la interpretación de los funcionarios nipones son los abultados aumentos en el presupuesto de defensa chino (desde el año 2007 a hoy China tiene el segundo presupuesto de defensa más alto del mundo, después de Estados Unidos. Japón se ubica en el octavo puesto y no ha realizado aumentos de envergadura en el mismo), el aumento de las incursiones de barcos chinos en las áreas cercanas a las islas Senkaku ${ }^{13}$, el aumento de actividades de la flota China en los mares circundantes a Japón, el hecho que desde 2010 China pasa a ser la segunda economía del mundo de acuerdo a su PBI, puesto que había ostentado Japón hasta ese momento. Como se puede observar, el ascenso de china en los asuntos mundiales hace que algunas de estas variables sean de naturaleza regional, mientras que otras de naturaleza internacional, como por ejemplo la fortaleza de la economía china.

Al contrario del caso chino, Corea del Norte sí es mencionada de manera directa como una amenaza. Por ejemplo, el Ministro de Defensa Nakatani, menciona en el Libro Blanco 2015 que el aumento de la capacidad en misiles balísticos y el desarrollo nuclear de Corea del Norte representan una amenaza seria e inminente para la seguridad de Japón (MOD, 2015) Además en los Libros Blancos siempre hay un capítulo dedicado a todo lo que acontece con Corea del Norte y en los correspondientes a 2015 y 2016 se menciona a dicho país como una amenaza. Por ejemplo, en el correspondiente al año 2015 se lee lo siguiente: "Such military trend in North Korea heightens tension in the Korean Peninsula and constitutes a serious destabilizing factor to the security not only of

malos cálculos, se han convertido en preocupaciones de seguridad para la región, incluyendo a Japón y a la comunidad internacional."

${ }^{10}$ El Libro Azul es un reporte de la Política Exterior nipona que recoge todas las actividades diplomáticas realizadas durante el año anterior. Por ejemplo, en este caso el Libro Azul del año 1992, reporta todas las actividades realizadas en el año 1991. Es publicado desde 1971, de manera anual, por el Ministerio de Asuntos Externos de Japón.

11 "[...] una serie de desarrollos militares en China, como se han visto en años recientes, levantan preocupación en la región y en la comunidad internacional en su conjunto."

12 "Hay preocupación dado que China está intentado alterar el status quo por la fuerza, antes que por vías legales."

${ }^{13}$ Las islas Senkaku están ubicadas en el Mar del Este de China y se encuentran en disputa entre Japón, China y Taiwán. Están deshabitadas y, según un informe de la ONU que data de 1968, poseen reservas de gas y petróleo. China y Taiwán argumentan que Japón usurpó las islas ilegalmente luego de la guerra sinojaponesa de 1895; mientras que Japón sostiene que las incorpora en 1895 por el principio res nullius del Derecho Internacional para territorios deshabitados. La soberanía sobre las islas para los 3 países es de importancia estratégica por el trazado de los espacios marítimos de jurisdicción alrededor de las mismas y por los recursos naturales que se encuentran en ellas. 
Japan but also of the entire region and the international community. Accordingly, it is necessary for Japan to pay utmost attention to them." ${ }^{14}$ (MOD, 2015)

No sólo en documentos oficiales encontramos referencias de Corea del Norte como amenaza, también en discursos del Primer Ministro Abe y del Ministro de Asuntos Externos Fumio Kishida. En una conferencia de prensa del Primer Ministro, en ocasión de la adopción de la nueva legislación de Septiembre de 2015, Abe se refirió a Corea del Norte de la siguiente manera: "Whether we like it or not, the security environment surrounding Japan is becoming increasingly severe. North Korea possesses several hundred ballistic missiles with a range that covers most of Japan's territory, and their development of nuclear weapons fitted to those missiles is becoming an ever more serious issue." ${ }^{15}$ (25 de Septiembre de 2015) Por otro lado, el Ministro Kishida, dejó en claro la posición de Japón con respecto a Corea del Norte en el Discurso de Política Exterior ante la Dieta en Febrero de 2015: "North Korea's continued nuclear and missile development poses a grave threat to peace and security in the region and in the international community as a whole" ${ }^{16}$ (12 de Febrero de 2015)

La percepción de Corea del Norte como amenaza por funcionarios japoneses es clara y proviene del ambiente regional. En este caso las variables externas tienen que ver con el lanzamiento de misiles balísticos norcoreanos al mar territorial de Japón, con los avances en el desarrollo nuclear militar; y también con el hecho de que desde los años 90 hasta el 2015 no se consiguieron avances en las negociaciones con Corea del Norte para que adopte un desarrollo nuclear pacífico. Por otro lado, no se puede dejar de mencionar que las relaciones entre ambos estados se vieron fuertemente deterioradas por el tema de los secuestros durante la Guerra Fría ${ }^{17}$, que Corea del Norte admitió en 2002. En 2014 Corea del Norte había accedido a establecer un Comité Especial de Investigación sobre los secuestros de ciudadanos japoneses, pero en 2016 decide disolverlo en represalia por las sanciones que Japón impuso a Corea del Norte por el lanzamiento de misiles. En el tiempo de duración del Comité, ningún reporte fue enviado al gobierno nipón sobre avances en las investigaciones.

Por último, además de las consideraciones acerca de China y Corea del Norte, ciertas consideraciones acerca de Estados Unidos motivaron la adopción de la nueva legislación en 2015. Por un lado, el avance de China a nivel mundial genera cuestionamientos a la posición de poder global de Estados Unidos, debilitando al principal aliado de Japón.

\footnotetext{
14 "Esta tendencia militar en Corea del Norte aumenta la tensión en la Península de Corea y constituye un factor de desestabilización serio para la seguridad no solo de Japón, sino también de toda la región y de la comunidad internacional."

15 “Nos guste o no, el ambiente de seguridad que rodea a Japón se está volviendo cada vez más severo. Corea del Norte posee cientos de misiles balísticos cuyo rango de alcance cubre la mayor parte del territorio japonés, y el desarrollo de armas nucleares que puedan ser incluidas en esos misiles se volvió un asunto de mayor seriedad."

16 "El continuo desarrollo nuclear y misilístico de Corea del Norte implica una grave amenaza para la paz y la seguridad de la región y del conjunto de la comunidad internacional."

${ }^{17}$ Doce ciudadanos japoneses fueron secuestrados durante la Guerra Fría por efectivos norcoreanos. En el 2002 el gobierno de Corea del Norte admitió los secuestros y además informó que 8 de las 12 personas secuestradas ya habían fallecido. De ahí en más comenzaron arduas negociaciones entre Japón y el régimen norcoreano por la vuelta de los que aún estaban vivos y la descendencia de aquellos que no habían logrado sobrevivir. Es un tema que aún no se ha resuelto.
} 
Por otro lado, recortes en el presupuesto de defensa norteamericano afectaron ciertos puntos de la alianza con Japón y generaron la duda de si Estados Unidos podría seguir haciéndose cargo de la defensa de Japón. De hecho, uno de los asesores de Abe en temas de seguridad, Yosuke Isozaki, se refirió al declinio norteamericano y la necesidad de reformar la defensa nipona: "Truth be told, the U.S. can no longer afford to play the world's policeman. [...] This is no longer an era when Japan is permitted to do nothing and count on America to protect us for free. It's become extremely important we do our own share alongside the U.S." ${ }^{18}$ (Hayashi, 2014)

La falta de reciprocidad de la alianza nipo-estadounidense no es un tema nuevo de discusión, pero debido a los cambios en el escenario internacional y en la región Asia Pacífico el tema volvió a tener relevancia. En las directrices para la alianza adoptadas a principios de 2015, se buscó que Japón tuviese mayores responsabilidades, por ejemplo ampliando las ocasiones en que las FAD pueden actuar. La nueva legislación del 2015 responde también a estas consideraciones acerca de la relación entre ambos estados. Teniendo en cuenta que las nuevas directrices venían negociándose desde 2013 y que su publicación es previa a la nueva legislación, algunos puntos de las directrices son recuperados en las dos leyes aprobadas. De hecho esto generó algunas controversias en Japón, porque la oposición al partido gobernante cuestionó que se aprobasen las directrices antes de que la legislación haya sido correctamente discutida y aprobada por la Dieta.

Una consideración interesante para completar el análisis de la relación Estados Unidos - Japón, lo trae a colación Saltzman (2015, p. 511): “Given the fact that Tokyo has no genuine alternative allies, and that the USA is the key pillar of Japanese national security, Japan is caught in a classical alliance dilemma-between abandonment (the ally defects during crisis) and entrapment (the ally forces involvement in crisis)" 19 Claramente, las modificaciones a la Política de Defensa nipona también apuntan a otorgar una mayor independencia de actuación a las FAD y a reducir el dilema descripto por Saltzman. Esto no significa que las relaciones entre ambos estados no sean buenas, ni que se esté considerando en romper con esa Alianza, sino que ambas partes han acordado otorgar mayores responsabilidades a Japón para reducir la falta de reciprocidad y que las FAD puedan involucrarse en mayores actividades relacionadas con la defensa de Japón.

La noción de amenaza en el caso estadounidense no es tan clara, pero sí se identifican algunas situaciones que generan preocupación para los funcionarios japoneses como el cambio en el balance de poder global y los recortes del presupuesto de defensa de Estados Unidos. Estas variables externas de naturaleza internacional, junto con las mencionadas para China y Corea del Norte, son las que motivaron la adopción de las nuevas leyes.

\footnotetext{
18 "La realidad es que Estados Unidos no puede afrontar más el rol de policía mundial. [...] Esta no es más una era en la cual se le permite a Japón hacer nada y contar de todas maneras con que Estados Unidos va a protegernos sin costo. Se ha vuelto extremadamente importante cumplir con nuestra parte, junto a Estados Unidos."

19 "Dado que Tokyo no tiene genuinos aliados alternativos y que USA es el pilar clave de la seguridad nacional de Japón, dicho país se ve atrapado en el clásico dilema de seguridad-entre el abandono (el aliado deserta durante una crisis) y la presión para actuar (el aliado obliga a participar en una crisis)."
} 


\section{Conclusiones}

El común denominador de estas situaciones es que han sido leídas por los funcionarios nipones como pérdidas de poder, como alteraciones en el status quo regional e internacional, y de ahí que sean percibidas como amenazas. El gobierno de Abe, a través de la ampliación de las situaciones en que las FAD puede actuar, busca balancear el ascenso de China en la región, busca mejorar sus capacidades de defensa contra Corea del Norte, busca avanzar en alcanzar la reciprocidad total en la alianza con Estados Unidos.

Estas modificaciones forman parte del proceso de normalización de la política de defensa nipona que comenzó con la creación de las FAD en la década de los 60, como se mencionó en la introducción. Los hitos más importantes en este proceso se dieron en 1992, cuando una ley autorizó a las FAD a salir del país para participar de Operaciones de Mantenimiento de la Paz de Naciones Unidas, y en 2003, cuando también a través de una legislación, las FAD participaron en la guerra en Irak realizando tareas de apoyo más que nada. Los ajustes realizados por Abe representan un paso más en este proceso y de ahí que no deban ser leídos como revolucionarios, sino como incrementales, como sostiene Liff $(2015$, p.81) "Since the end of the Cold War, Japan's leaders have made incremental changes designed to ensure that the JSDF and U.S.-Japan alliance adapt to changing regional and global threats." ${ }^{20}$ Es importante rescatar esto, dado que muchas veces se han tomado los ajustes realizados como hechos aislados y como parte de una estrategia de Japón y sobre todo del gobierno de Abe de volver al país asiático al militarismo del siglo pasado. Los ajustes introducidos están basados en el pacifismo de la constitución y han permitido que Japón participe con sus FAD en numerosas iniciativas de seguridad internacional comandadas por la ONU, como así también en numerosas tareas de ayuda humanitaria y de asistencia ante desastres naturales.

\section{Bibliografía}

Akimoto, D. (2012), "Positive Pacifism" and the development of Japan's PKO Policy", Soka University Peace Research Institute, $\mathrm{n}^{\circ} 26, \mathrm{pp} .27-53$.

Álvarez Calzada, J. O. (2004), "La reforma constitucional en Japón como una oportunidad, no una amenaza", Documentos CIDOB, serie Asia, $\mathrm{n}^{\circ} 7$.

Andhika Prajuli, W. y Pariwita, N. A. (2015), “Between fear and hope on Japan's new defence policy", East Asia Forum.

Arteaga, F. (2007), "Japón y su nueva política de seguridad internacional", Real Instituto Elcano, $n^{\circ} 41 / 2007$.

Auslin, M. (2016), “Japan's new realism”, Foreign Affairs, edición marzo/abril.

Baker, G. y Nishiyama, G. (2013), “Abe Says Japan Ready to Counter China's Power”, Wall Street Journal.

\footnotetext{
20 "Desde el fin de la Guerra Fría, los líderes japoneses han realizado cambios incrementales, diseñados para asegurar que las FAD y la alianza Estados Unidos - Japón se adapten a las cambiantes amenazas regionales y globales."
} 
Barcelona Centre for International Affairs (CIDOB), (2013), "La Política Exterior de Japón", En CIDOB (2013), Anuario Internacional CIDOB 2013, pp. 401-408.

(2013), "Política de seguridad y defensa de Japón", en CIDOB (2013), Anuario Internacional CIDOB 2013, pp. 409-419.

Berkshire Miller, J. (2014a), "Battle-Ready Japan? The Real Story Behind Tokyo's First National Security Strategy", Foreign Affairs, edición enero. (2014b), “How will Japan's new NSC work?”, The diplomat.

Feaver, P. D. y otros (2000), "Correspondence. Brother can you spare a paradigm? (Or was anybody ever a realist?)", International Security, v. 25, n 1, pp. 165-193.

Funaiole, M. P. (2015), “Conceptualizing Japan's Foreign Policy trajectory through social identity theory", East Asia, v. 32, n² 4, pp. 361-383.

Hayashi, Y. (2014), "Japanese Officials Discuss Perceived Decline in U.S. Military Might", Wall Street Journal.

International Institute For Strategic Studies (IISS), (2016), "'Proactive Pacifism': the new normal in Japan's foreign and security policy", en IISS (2016), Asia-Pacific Regional Security Assessment 2016, Londres: International Institute for Strategic Studies, pp.39-54.

(2017) The Military Balance. Londres: International Institute for Strategic Studies.

Inoguchi, T. (2014), "A call for a new Japanese foreign policy: the dilemmas of a stakeholder state", International Affairs, v. 90, n 4, pp. 943-958.

Jimbo, K. (2013), "Política exterior japonesa: 2006-2013. Una respuesta al ascenso de China", Anuario Internacional CIDOB, pp.349-356.

Liff, A. P. (2015), "Japan's Defense Policy: Abe the evolutionary", The Washington Quarterly, v. $38, n^{\circ} 2$, pp. 79-99.

Lobell, S. E. (2009), Threat assessment, the state and Foreign Policy: a neoclassical realist model, en Lobell, S. E.; Ripsman, N. M. y Taliaferro, J. W. (2009), Neoclassical realism, the State and Foreign Policy, New York: Cambridge University Press, pp.42-74.

López I Vidal, L. (2007), “La nueva estrategia de seguridad japonesa: La normalización de su diplomacia”, Anuario Asia Pacífico 2006, CIDOB, pp. 191-204.

Ministry of Defense (MOD), (2016), Defense of Japan 2016, Tokyo: Ministry of Defense. (2015), Defense of Japan 2015, Tokyo: Ministry of Defense.

(2015), The Guidelines for Japan-U.S. Defense Cooperation, Tokyo: Ministry of Defense. (2014), Defense of Japan, Tokyo: Ministry of Defense. (2013 a), National Security Strategy, Tokyo: Ministry of Defense. (2013 b), National Defense Program Outlines, Tokyo: Ministry of Defense. 
Ministry of Foreign Affairs (MOFA), (2016a), Diplomatic Bluebook 2016, Tokyo: Ministry of Foreign Affairs.

(2016b), National Security Council (NSC), disponible online en https://www.mofa.go.jp/fp/nsp/page1we 000080.html, consultado el 02/07/2018.

(2015), Outline of Japan's International Peace Cooperation, disponible online en: https://www.mofa.go.jp/fp/ipc/page22e 000683.html, consultado el 2/07/2018.

(2014), Cabinet Decision on Development of Seamless Security Legislation to Ensure Japan's Survival and Protect its People, disponible online en: https://www.mofa.go.jp/fp/nsp/page23e 000273.html , consultado el 02/07/2018.

Murakami, Y. (2011), “Asia del Este y la política exterior del Japón: desafíos para el siglo XXI", Agenda Internacional, t. XVIII, n²29, pp. 19-54.

Pereira Britesi, P. V. y Prates Cattelan, P. H. (2016), "A política externa japonesa de Shinzo Abe: revolução silenciosa?”, Boletim de Conjuntura NERINT, v. 1, n³, pp. 1624.

Pérez Javier, M. (2015), “Japón y China (1993-2013): Espacios de confrontación e interacción", Observatorio Iberoamericano de la Economía y la Sociedad del Japón, v. $7, n^{\circ} 24$.

Ríos, X. (2016), "El conflicto China-Japón”, Observatorio Iberoamericano de la Economía y la Sociedad del Japón, v. 8, n² 25.

(2013), "Las crisis en los mares de China: implicaciones geopolíticas y en materia de seguridad”, en INSTITUTO ESPAÑOL DE ESTUDIOS ESTRATÉGICOS (IEEE), (2013), Panorama Estratégico, Madrid: Ministerio de Defensa, pp.139-167.

Rodríguez Asien, E. (2015), “Japón y el polémico artículo 9", Observatorio de la Economía y la Sociedad del Japón, v. 7, n² 24.

Rose, G. (1998), "Neoclassical Realism and Theories of Foreign Policy", World Politics, v. 51, $\mathrm{n}^{\circ} 1$, pp. 144-172.

Saltzman, I. Z. (2015), “Growing Pains: Neoclassical Realism and Japan's Security Policy Emancipation”, Contemporary Security Policy, v. 36, n³ 3, pp. 498-527.

SANG-HUN, C. (2016), “North Korea Cancels Investigation Into Abductions of Japanese Citizens", The New York Times. 